\title{
Video Article \\ Avian Influenza Surveillance with FTA Cards: Field Methods, Biosafety, and Transportation Issues Solved
}

\author{
Robert H.S. Kraus ${ }^{1}$, Pim van Hooft ${ }^{1}$, Jonas Waldenström ${ }^{2}$, Neus Latorre-Margalef ${ }^{2}$, Ronald C. Ydenberg ${ }^{1,3}$, Herbert H.T. Prins ${ }^{1}$ \\ ${ }^{1}$ Resource Ecology Group, Wageningen University \\ ${ }^{2}$ Section for Zoonotic Ecology and Epidemiology, School of Natural Sciences, Linnaeus University \\ ${ }^{3}$ Centre for Wildlife Ecology, Simon Fraser University
}

Correspondence to: Robert H.S. Kraus at robert.kraus@senckenberg.de

URL: https://www.jove.com/video/2832

DOI: doi:10.3791/2832

Keywords: Immunology, Issue 54, AI, Influenza A Virus, zoonoses, reverse transcription PCR, viral RNA, surveillance, duck trap, RNA preservation and storage, infection, mallard

Date Published: 8/2/2011

Citation: Kraus, R.H., van Hooft, P., Waldenström, J., Latorre-Margalef, N., Ydenberg, R.C., Prins, H.H. Avian Influenza Surveillance with FTA Cards: Field Methods, Biosafety, and Transportation Issues Solved. J. Vis. Exp. (54), e2832, doi:10.3791/2832 (2011).

\section{Abstract}

Avian Influenza Viruses (AIVs) infect many mammals, including humans ${ }^{1}$. These AIVs are diverse in their natural hosts, harboring almost al possible viral subtypes ${ }^{2}$. Human pandemics of flu originally stem from AIVs ${ }^{3}$. Many fatal human cases during the H5N1 outbreaks in recent years were reported. Lately, a new AIV related strain swept through the human population, causing the 'swine flu epidemic' ${ }^{4}$. Although human trading and transportation activity seems to be responsible for the spread of highly pathogenic strains ${ }^{5}$, dispersal can also partly be attributed to wild birds $^{6,7}$. However, the actual reservoir of all AIV strains is wild birds.

In reaction to this and in face of severe commercial losses in the poultry industry, large surveillance programs have been implemented globally to collect information on the ecology of AIVs, and to install early warning systems to detect certain highly pathogenic strains ${ }^{8-12}$. Traditional virological methods require viruses to be intact and cultivated before analysis. This necessitates strict cold chains with deep freezers and heavy biosafety procedures to be in place during transport. Long-term surveillance is therefore usually restricted to a few field stations close to well equipped laboratories. Remote areas cannot be sampled unless logistically cumbersome procedures are implemented. These problems have been recognised ${ }^{13,14}$ and the use of alternative storage and transport strategies investigated (alcohols or guanidine) ${ }^{15-17}$. Recently, Kraus et al. ${ }^{18}$ introduced a method to collect, store and transport AIV samples, based on a special filter paper. FTA cards ${ }^{19}$ preserve RNA on a dry storage basis $^{20}$ and render pathogens inactive upon contact ${ }^{21}$. This study showed that FTA cards can be used to detect AIV RNA in reverse-transcription PCR and that the resulting cDNA could be sequenced and virus genes and determined.

In the study of Kraus et al. ${ }^{18}$ a laboratory isolate of AIV was used, and samples were handled individually. In the extension presented here, faecal samples from wild birds from the duck trap at the Ottenby Bird Observatory (SE Sweden) were tested directly to illustrate the usefulness of the methods under field conditions. Catching of ducks and sample collection by cloacal swabs is demonstrated. The current protocol includes upscaling of the work flow from single tube handling to a 96-well design. Although less sensitive than the traditional methods, the method of FTA cards provides an excellent supplement to large surveillance schemes. It allows collection and analysis of samples from anywhere in the world, without the need to maintaining a cool chain or safety regulations with respect to shipping of hazardous reagents, such as alcohol or guanidine.

\section{Video Link}

The video component of this article can be found at https://www.jove.com/video/2832/

\section{Duck Trapping and Cloacal Swabbing}

1. Trap dabbling ducks of the genus Anas (or other birds) in a cage by attracting them with lure ducks or food, and put them into individual card board boxes. Transportation time in the box should be kept to a minimum, for instance, by installing a field station within a short distance to the trap. Logistic circumstances may vary in each study. The advice and approval of the relevant animal ethics committee needs to be sought for each new set-up. Alternatively, also hunter shot birds can be sampled.

2. Take the duck out of the box by holding its wings tight to its body. Sample a fresh dropping, which will be present in most of the cases, directly from the bottom of the box. Pick them up with a sterile swab and apply the fluid to a Whatman FTA card. If not, perform cloacal swabbing.

3. Turn the duck on its back. This permits access to the cloaca and facilitates sampling. The cloaca is a protruding structure situated below the abdomen, close to the base of the tail. An experienced person could hold and sample the bird at the same time, or else a second person can assist. 
4. Carefully insert the sterile plastic rayon swab (Copan, Italy) approximately $1 \mathrm{~cm}$ and make a gentle swirl of the cloaca. Roll the swab with the fluids from the cloaca over the surface of a Whatman FTA card. After sampling has been performed immediate release of the animal is desireable.

5. Dry the FTA cards at room temperature for at least 1 hour, then store each sample individually in paper bags (e.g. envelopes). Storage is possible at room temperature, possibly with silica beads in humid climates. The sending and receiving institutions' biosafety officers can permit to send FTA cards by regular mail, since the pathogens become inactive upon contact with the FTA card surface.

\section{Viral RNA Isolation}

1. Extract the FTA card material including faeces/cloacal fluid. Punch three discs with a Harris $2 \mathrm{~mm}$ puncher and place all of those into a well of an RNase free 96well plate. Between each new sample, clean the puncher carefully with alcohol and a Kim precision wipe (Kimtech). Always use positive and negative controls. A positive control can consist of a laboratory strain freshly applied to an FTA card, or a natural sample which is know to yield a positive result. As negative control, punch out discs from an empty FTA card. Additionally, leave another well free for a PCR control later in your experiment (with water as template).

2. Add $70 \mu \mathrm{l}$ RNA rapid extraction solution (Ambion) and heat-seal the plate (AbGene Thermo-Sealer). Incubate 5 minutes on a plate shaker at room temperature with the determined speed that does not cause spill-over (this depends on the used plate shaker model; test in advance).

3. Carry viral RNA isolation according to the manufacturer's protocols with the MagMAX-96 viral RNA isolation kit (Ambion). In brief: Add 130 $\mu$ prepared lysis/binding solution (from the kit) to each well. Transfer $50 \mu$ l extracted FTA card material to each well. Shake plate for 1 minute.

4. Add $20 \mu \mathrm{l}$ prepared bead mix (from the kit) to each sample and mix by pipetting up and down. Shake on determined speed for 5 minutes. RNA molecules will bind to the magnetic beads.

5. Move the plate to a magnetic 96-well stand to capture the beads. Depending on the model of magnetic stand used, this can take more than 5 minutes. When the solution has turned completely clear, remove and discard the supernatant. Then remove the plate from the magnetic stand.

6. Wash the beads twice with wash solution 1 and twice with wash solution 2 (from the kit). For each of the 4 wash steps add $150 \mu$ l prepared wash solution to each sample, shake for 1 minute on determined speed, move the plate to the magnetic stand, capture beads for about 5 minutes (or until the solution is completely clear), remove and discard the supernatant. Then remove the plate from the magnetic stand, add the next wash solution, and carry out the washing steps as previously. After the last washing step, remove as much wash solution as possible and air dry the beads at room temperature in the plate shaker for 2 minutes.

7. Add $50 \mu$ l of elution buffer (from the kit) to release RNA from the beads and shake for 4 minutes on the plate shaker. Capture beads as previously described. The supernatant now contains the isolated RNA which is ready for downstream applications.

\section{RT-PCR of the AIV Matrix Gene}

Carry out reverse transcription PCR (RT-PCR) with the One-Step Access RT-PCR system (Promega) in $25 \mu$ reactions (adjusted from Kraus et al. ${ }^{18}$ and Fouchier et $a .^{22}$ ):

\begin{tabular}{|l|l|}
\hline Nuclease free water & $1.5 \mu \mathrm{l}$ \\
\hline AMVITfl5 buffer & $5 \mu \mathrm{l}$ \\
\hline dNTPs & $0.5 \mu \mathrm{l}$ \\
\hline primer M52C $22(10 \mu \mathrm{M})$ & $2.5 \mu \mathrm{l}$ \\
\hline primer M253R $^{22}(10 \mu \mathrm{M})$ & $2.5 \mu \mathrm{l}$ \\
\hline $\mathrm{MgSO}_{4}(25 \mathrm{mM})$ & $7 \mu \mathrm{l}$ \\
\hline AMV reverse transcriptase $\left.(5 \mathrm{u} / \mu \mathrm{l})^{\mathrm{N}}\right)$ & $0.5 \mu \mathrm{l}$ \\
\hline Tfl polymerase $(5 \mathrm{u} / \mu \mathrm{l})$ & $0.5 \mu \mathrm{l}$ \\
\hline RNA sample & $5 \mu \mathrm{l}$ \\
\hline
\end{tabular}

PCR conditions in a Biometra T1 thermocycler are: initial reverse transcription of 45 minutes at $45^{\circ} \mathrm{C}$, followed by 2 minutes initial denaturation at $94^{\circ} \mathrm{C}$ and 40 cycles of: $94^{\circ} \mathrm{C}$ for 1 minute, $56^{\circ} \mathrm{C}$ for 1 minute, and $68^{\circ} \mathrm{C}$ for 2 minutes. An additional 7 minutes elongation at $68^{\circ} \mathrm{C}$ concludes the amplification.

\section{Screening for Al-positive Samples and Purification of Targeted Fragments from Gel}

1. Load $2 \mu \mathrm{l}$ of the PCR product mixed with $2 \mu \mathrm{l} 5 \mathrm{x}$ loading dye (BioRad) and $6 \mu \mathrm{l}$ water on a $1 \%$ agarose gel (Roche) stained with $1 \%$ ethidium bromide $(2.5 \mu \mathrm{l}$ per $100 \mathrm{ml}$ gel) for a pre-screening.

2. Run the gel for 1 hour at $120 \mathrm{~V}$, along with a DNA size standard (BioRad EZ load $100 \mathrm{bp}$ ladder), visualise with a gel documentation system. See an example in Figure 1.

3. Select samples with amplified fragments in the expected size range (between $200 \mathrm{bp}$ and $300 \mathrm{bp}$ (target fragment is $244 \mathrm{bp}$ ). Load the whole PCR reaction volume (of which $\sim 23 \mu \mathrm{l}$ are left) of these candidates with $6 \mu \mathrm{l} 5 \mathrm{x}$ loading dye on a $2 \%$ ethidium bromide stained agarose gel and run for 2 hours.

4. Place the gel on a UV-transilluminator (Bioblock Scientific) and inspected visually. See an example in Figure 2. Excise bands of the correct size from gel with a scalpel and placed into individual $1.5 \mathrm{ml}$ reaction tubes. Purify fragments from gel, for instance with the Zymoclean Gel DNA Recovery Kit (Zymo Research). 


\section{Sequencing and Identification of PCR Products}

1. Carry out Sanger sequencing of the target fragments, for instance on an $A B I 3730$ capillary sequencer with $A B I$ Big Dye 3.1 chemistry

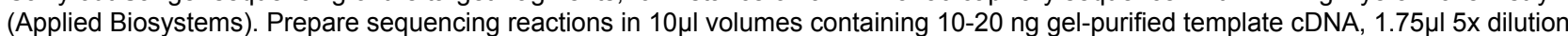
buffer, $0.5 \mu \mathrm{l}$ Big Dye V3.1 premix, $1 \mu \mathrm{l}$ forward primer $\left(\mathrm{M} 52 \mathrm{C}^{20}, 10 \mathrm{mM}\right)$, and ddH2O. Cycling conditions are: 1 min initial denaturation, followed by 25 cycles of: $10 \mathrm{~s}$ at $96^{\circ} \mathrm{C}, 5 \mathrm{~s}$ at $45^{\circ} \mathrm{C}$ and $4 \mathrm{~min}$ at $60^{\circ} \mathrm{C}$. Purify and prepare the sequencing reaction according to your internal protocols.

2. Identify resulting cDNA sequences against nucleotide databases such as GenBank23, e.g. by web based tools such as BLAST at the National Centre for Biotechnology Information (NCBI, http://blast.ncbi.nlm.nih.gov/Blast.cgi).

\section{Representative Results:}

Mallards (Anas platyrhynchos) were sampled at Ottenby Bird Observatory in December 2007. From each mallard a sample on FTA card was taken as described in this protocol. After shipping, the FTA cards were kept in a freezer at $-20^{\circ} \mathrm{C}$ for two years. The same FTA card sample of the laboratory isolate tested in Kraus et al. ${ }^{18}$ was included as positive control, as well as nine tenfold serial dilutions of it. Two negative controls were i) extraction from an empty FTA card, to test if there was carry-over from the puncher, and ii) RT-PCR reaction in which nuclease free water was used as template, to test if contamination occurred during, or in preparation of the PCR reaction.

84 samples were analysed. A gel picture of the PCR products from these 84 samples can be found in Figure 1. From natural samples a multitude of unspecific bands can be observed due to the presence of various microbial contaminations in the faeces. However, the target fragment of the primer pair is $244 \mathrm{bp}$ long. The whole PCR-reaction volume of a subset of the samples which produced fragments in approximately the correct size range (between $200 \mathrm{bp}$ and $300 \mathrm{bp}$ ) was loaded on gel (Figure 1). An illustration of which of the bands were cut from the gel can be found in Supplementary Figure 1. In addition to the positive control, two of these samples (69899 and 69912) were positive by the new protocol. A BLAST search against the NCBI nucleotide database revealed their identity as AI matrix gene (Figure 3), while all the other bands resembled bacterial sequences most closely, or did not yield a readable sequence at all.

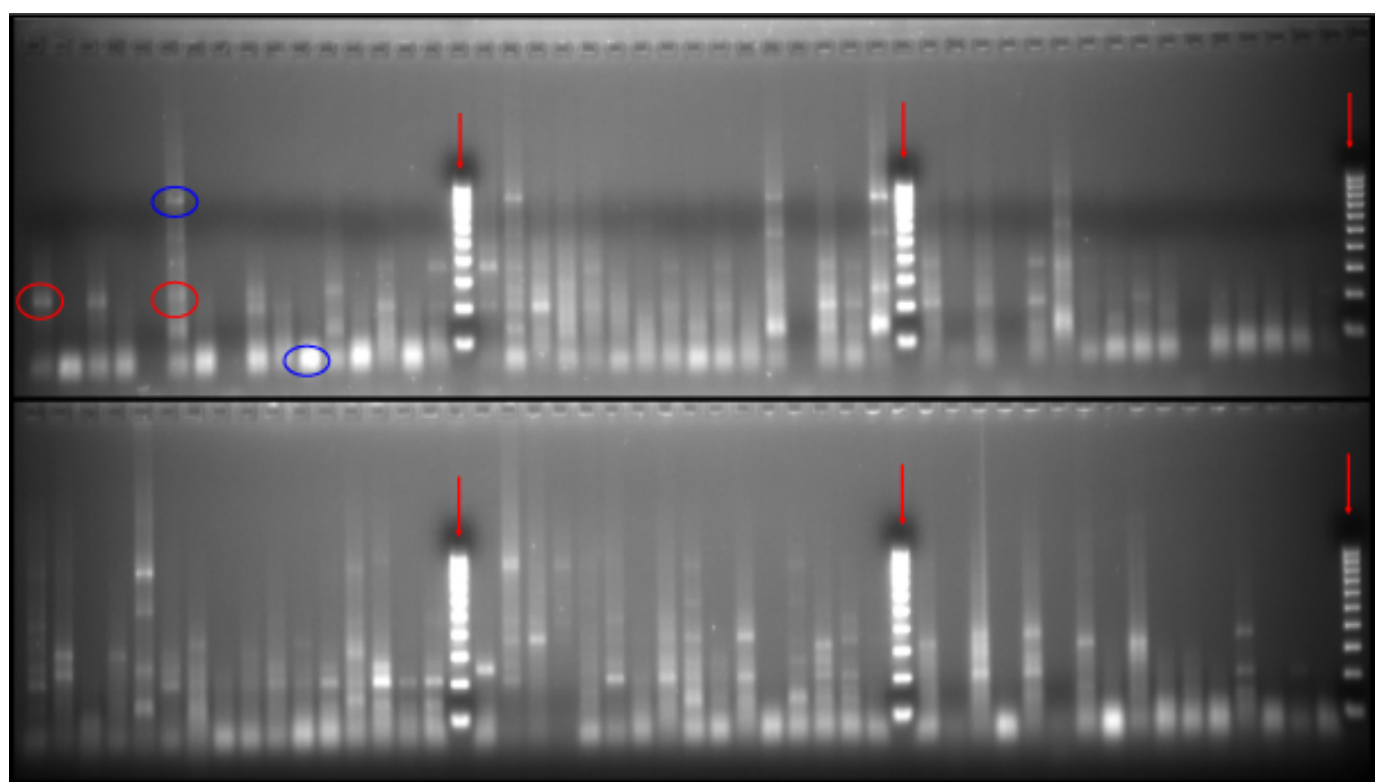

Figure 1. Gel picture of a preliminary screening of the PCR products. $2 \mu \mathrm{IPCR}$ product of positive control, serial dilutions of the positive control, two negative controls and 84 cloacal samples were loaded. 48 samples are shown in the top gel panel, and 48 samples in the bottom panel. Red arrows indicate gel lanes used for $100 \mathrm{bp}$ DNA size standard. For illustration, red circles show bands in the expected size range. Blue circles indicate an unspecific amplicon (top left) or a primer dimer artefact below $100 \mathrm{bp}$ in size (bottom right). 


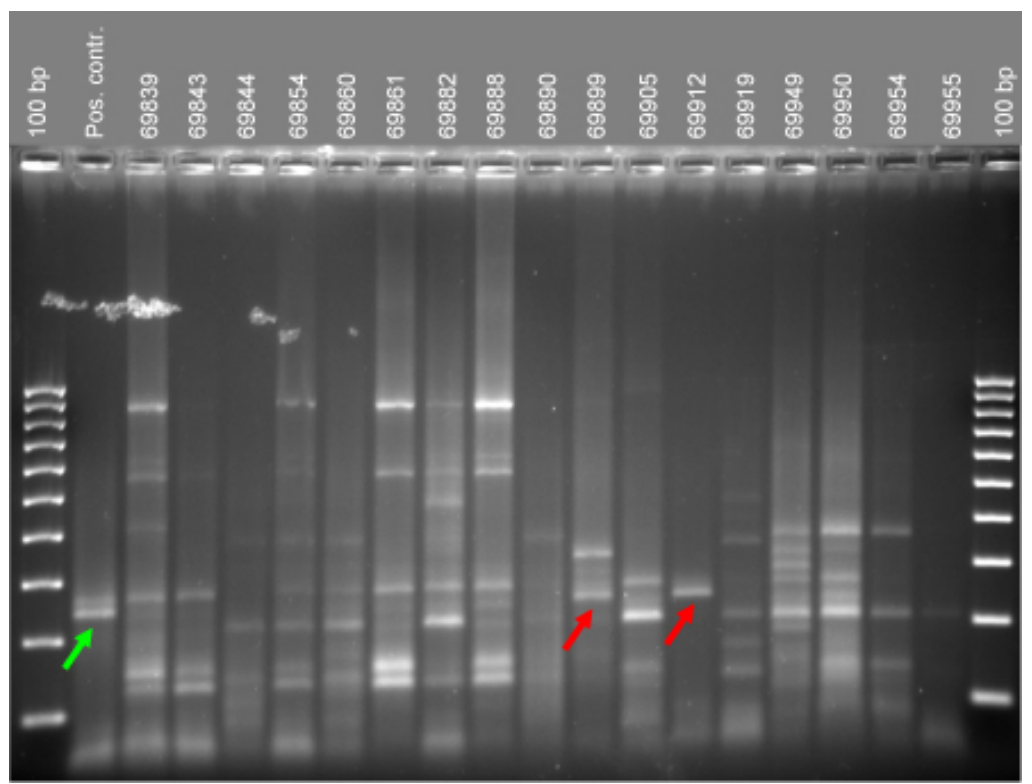

Figure 2. Selection of samples with fragments in the correct size range. Samples with bands between $200 \mathrm{bp}$ and $300 \mathrm{bp}$ (target fragment $244 \mathrm{bp}$ ) were chosen. The green arrow indicates the positive control, the two red arrows indicate samples which were confirmed to be AIV positive by comparing their CDNA sequences to the NCBI nucleotide database.

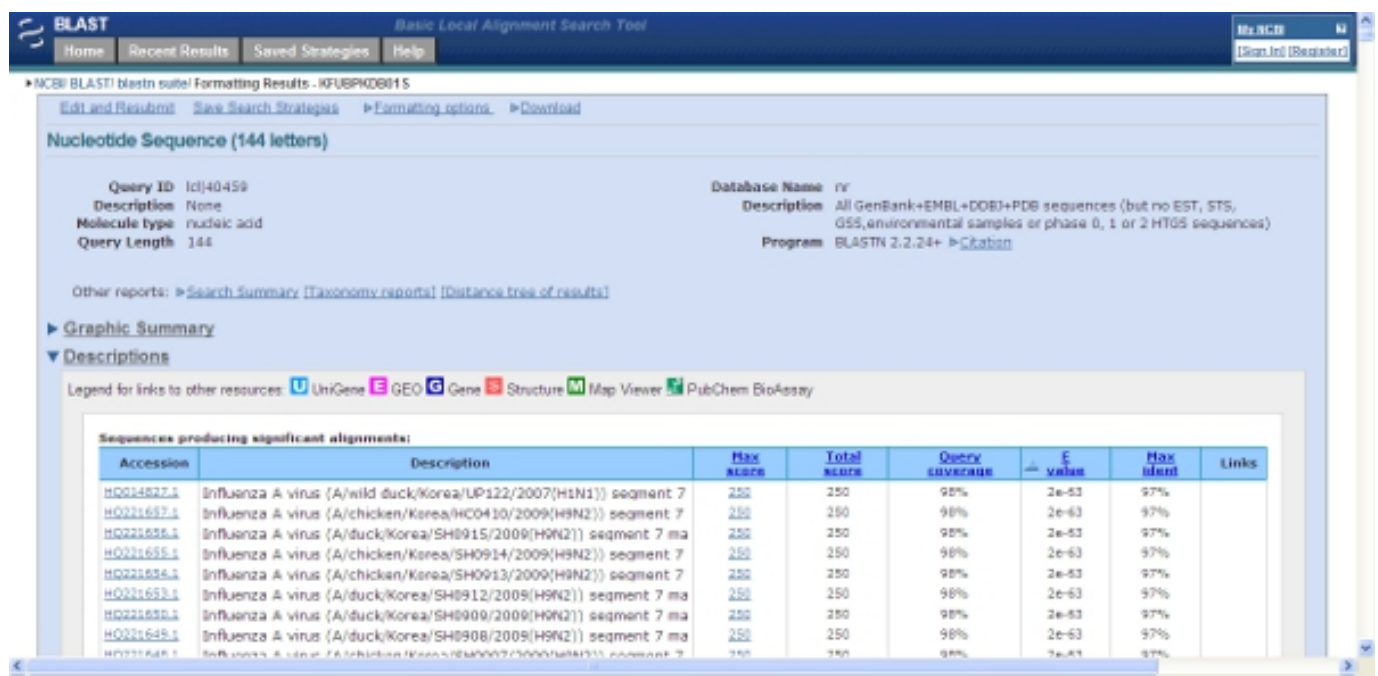

Figure 3. Screen capture of a representative BLAST search at NCBI. One of the CDNA sequences obtained from the excised fragments was queried against the nucleotide database at the NCBI website. The sample is correctly identified as Al Matrix gene fragment. To view a full sized version of this image, click here. 


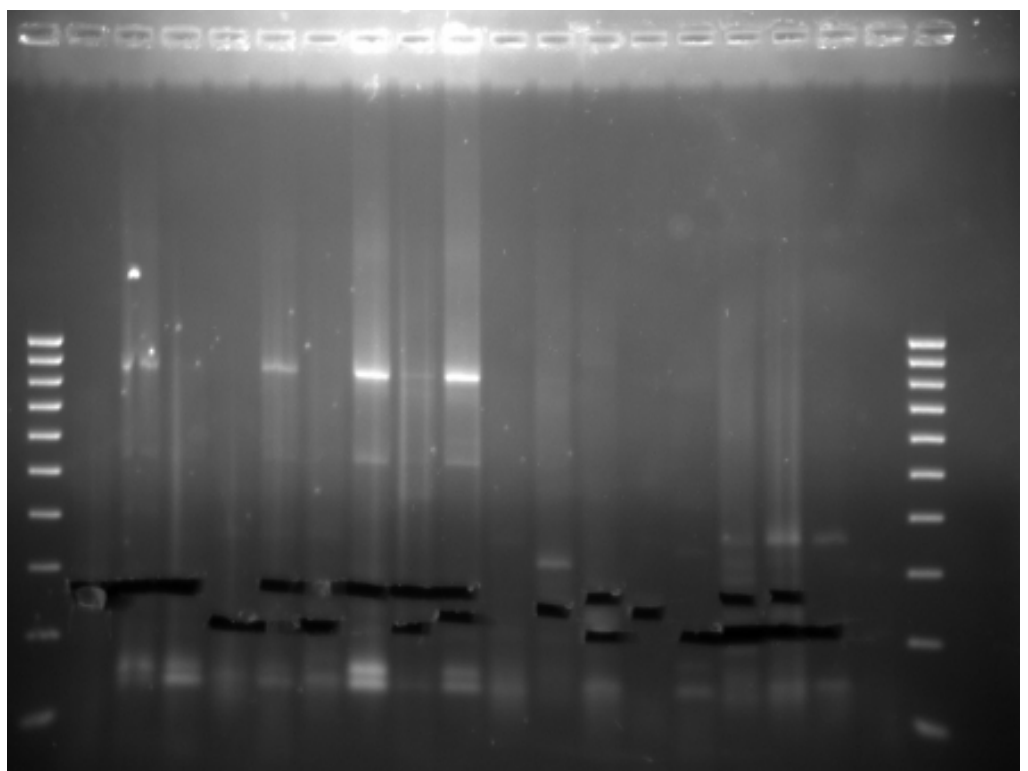

Supplementary Figure S1. Bands of selected samples excised from gel. This picture was taken from the gel depicted in Figure 1 after candidate bands between $200 \mathrm{bp}$ and 300 bp were cut out.

\section{Discussion}

The protocol described here provides a supplementary method to screen faecal or similar samples for the presence of AIV. It was especially designed to make sample collection quick and easy. This makes it possible for less trained persons, such as hunters or wildlife managers, to contribute to Al surveillance. No cool chains need to be applied, although freezing the samples is recommended where possible. A few days of room temperature, for instance during transport to the laboratory, were not a problem for RNA molecules on FTA cards, as long as the cards remained dry. Other non-cooled storage systems that are currently evaluated by the research community, such as alcohol ${ }^{15}$ or guanidine ${ }^{16}$, require special shipping arrangements because of their hazardous nature. In contrast, the FTA card method does not require shipping of hazardous materials. However, another interesting storage medium in this respect is RNAlater ${ }^{\text {TM }}$ that is not hazardous, either ${ }^{17}$. Sample analysis can be carried out in any standard molecular laboratory. No special equipment other than for usual PCR reactions and capillary sequencing was needed. All steps could be carried out without a biosafety level because already at sample collection the potential pathogenic agents were inactivated by the antibacterial and antiviral activity of the FTA card.

Cross-contamination and subsequent false positive samples were not observed in our trials with wild bird samples. However, when working with RNA and PCR it is always advisable to pay special attention to clean working places and separate rooms for pre- and post-PCR steps. Working in a fume hood decreases the risk of aerosol contamination in the laboratory. Pipetting needs to be carried out with filter tips.

From a previous study on samples taken simultaneously from the same ducks we know that six of the 84 samples were positive by the traditional RealTime RT-PCR method ${ }^{24}$ and the Ct values from RealTime RT-PCR are known. The two positive samples detected by our method stem from ducks which had $\mathrm{Ct}$ values $<30$ (indicating a high concentration of viral RNA). The other four samples which were positive with the traditional protocol had $\mathrm{Ct}$ values $>30$ (less concentrated) and could not be detected by our protocol.

Only samples with relatively high virus titers were positive in our assay and it is likely that sensitivity of the method was the source of failure to detect all positive samples. Further, the sample size in the current study was very low and the method can be completely developed and assessed if more controlled and rigorous experiments are carried out. However, if these samples would have been collected from a remote area, traditional analysis would not have been possible at all. Additionally, these first results stem from pilot experiments which need further optimisation. A period of two years storage in a regular $-20^{\circ} \mathrm{C}$ freezer after sample collection probably also affected the quality of the viral RNA. This possible RNA degradation is an important issue when dealing with room temperature storage of inactivated viruses. Samples stored in alternative liquids as mentioned above suffer from significant degradation which impacts analysis of longer stretches of the viral genome ${ }^{16}$. Although not tested in our study, FTA cards have proven to be well suited to preserve intact RNA molecules in other RNA systems that are very similar to Avian Influenza viruses ${ }^{25,26}$.

\section{Disclosures}

Trapping, handling and sampling of birds were done following national legislation and were approved by the Swedish Board of Agriculture and the research animal ethics committee.

\section{Acknowledgements}

We thank Bert Dibbits for technical assistance. The Animal Breeding and Genomics Group, Wageningen University, The Netherlands, generously hosted us in their laboratory. The personnel of the Ottenby Bird Observatory, Sweden, is thanked for trapping and sampling the mallards, in particular Magnus Hellström, Marcus Danielsson, Christopher Magnusson and Stina Andersson. We thank Sanne Svensson, Jonatan Qvist and 
Per-Axel Gjöres for filming at Ottenby, and Mano Camon for filming in the lab. Daniel Bengtson provided beautiful duck photographs for the video portion of this publication. Further free material from the CDC Public Health Image Library (PHIL; http://phil.cdc.gov/phil/) was used: The electron micrograph (no. 280; by Dr. Erskine Palmer) and illustration (no. 11823; by Douglas Jordan) of the influenza virus. Financial support was given by the KNJV (Royal Netherlands Hunters Association), the Dutch Ministry of Agriculture, the Faunafonds and the Stichting de Eik Trusts (both in The Netherlands), the Swedish Research Council (grant no. 2007-20774) and the EC-founded Newflubird project. RNA isolation chemicals were a generous gift of Ambion, Inc, the RNA company. This is contribution No. 245 from the Ottenby Bird Observatory.

\section{References}

1. Pulido, F. The genetics and evolution of avian migration. BioScience. 57, 165-174 (2007)

2. Bin Muzaffar, S., Ydenberg, R.C., \& Jones, I.L. Avian influenza: An ecological and evolutionary perspective for waterbird scientists. Waterbirds. 29, 243-257 (2006).

3. Taubenberger, J. K. The origin and virulence of the 1918 "Spanish" influenza virus. Proc. Am. Philos. Soc. 150, 86-112 (2006)

4. Butler, D. Swine flu goes global. Nature. 458, 1082-1083 (2009).

5. Normile, D. Avian influenza. Wild birds only partly to blame in spreading H5N1. Science. 312, 1451 (2006).

6. Si, Y. et al. Spatio-temporal dynamics of global H5N1 outbreaks match bird migration patterns. Geospat. Health. 4, 65-78 (2009).

7. $\mathrm{Si}$, Y. et al. Environmental factors influencing the spread of the highly pathogenic avian influenza H5N1 virus in wild birds in Europe. Ecol. Soc. 15, $26(2010)$

8. Chen, H. et al. Properties and dissemination of H5N1 viruses isolated during an influenza outbreak in migratory waterfowl in Western China. J. Virol. 80, 5976-5983 (2006).

9. Gaidet, N. et al. Avian influenza viruses in water birds. Africa. Emerg. Infect. Dis. 13, 626-629 (2007).

10. Krauss, S. et al. Influenza in migratory birds and evidence of limited intercontinental virus exchange. PLoS Pathog. 3, e167 (2007).

11. Parmley, E. J. et al. Wild bird influenza survey, Canada, 2005. Emerg. Infect. Dis. 14, 84-87 (2008).

12. Wallensten, A. et al. Surveillance of influenza A virus in migratory waterfowl in northern Europe. Emerg. Infect. Dis. 13, 404-411 (2007).

13. Munster, V.J. et al. Practical considerations for high-throughput influenza A virus surveillance studies of wild birds by use of molecular diagnostic tests. J. Clin. Microbiol. 47, 666-673 (2009).

14. Latorre-Margalef, N. et al. Effects of influenza A virus infection on migrating mallard ducks. Proc. R. Soc. B. 276, 1029-1036 (2009)

15. Runstadler, J.A. et al. Using RRT-PCR analysis and virus isolation to determine the prevalence of avian influenza virus infections in ducks at Minto Flats State Game Refuge, Alaska, during August 2005. Arch. Virol. 152, 1901-1910 (2007).

16. Evers, D. L., Slemons, R. D. \& Taubenberger, J. K. Effect of preservative on recoverable RT-PCR amplicon length from influenza A virus in bird feces. Avian Dis. 51, 965-968 (2007).

17. Forster, J.L., Harkin, V.B., Graham, D.A., \& McCullough, S.J. The effect of sample type, temperature and RNAlater (TM) on the stability of avian influenza virus RNA. J. Virol. Methods 149, 190-194 (2008).

18. Kraus, R.H.S. et al. Avian influenza surveillance: On the usability of FTA cards to solve biosafety and transport issues. Wildfowl, 215-223 (2009).

19. Smith, L.M. \& Burgoyne, L. A. Collecting, archiving and processing DNA from wildlife samples using FTA databasing paper. BMC Ecol. 4,4 (2004).

20. Rogers, C.D.G. \& Burgoyne, L. A. Reverse transcription of an RNA genome from databasing paper (FTA). Biotechnol. Appl. Biochem. 31, 219-224 (2000).

21. Rogers, C. \& Burgoyne, L. Bacterial typing: Storing and processing of stabilized reference bacteria for polymerase chain reaction without preparing DNA - An example of an automatable procedure. Anal. Biochem. 247, 223-227 (1997).

22. Fouchier, R.A.M. et al. Detection of influenza a viruses from different species by PCR amplification of conserved sequences in the matrix gene. J. Clin. Microbiol. 38, 4096-4101 (2000).

23. Benson, D.A., Karsch-Mizrachi, I., Lipman, D.J., Ostell, J., \& Sayers, E.W. GenBank. Nucleic Acids Res. 38, D46-D51 (2009).

24. Spackman, E. et al. Development of a real-time reverse transcriptase $\mathrm{PCR}$ assay for type A influenza virus and the avian $\mathrm{H} 5$ and $\mathrm{H} 7$ hemagglutinin subtypes. J. Clin. Microbiol. 40, 3256-3260 (2002).

25. Muthukrishnan, M., Singanallur, N.B., Ralla, K., \& Villuppanoor, S.A. Evaluation of FTA cards as a laboratory and field sampling device for the detection of foot-and-mouth disease virus and serotyping by RT-PCR and real-time RT-PCR. J. Virol. Methods 151, 311-316 (2008).

26. Perozo, F., Villegas, P., Estevez, C., Alvarado, I., \& Purvis, L.B. Use of FTA filter paper for the molecular detection of Newcastle disease virus. Avian Pathol. 35, 93-98 (2006). 